\title{
Amphibious Surveillance Robot with Smart Sensor Nodes
}

\author{
V. Divya ${ }^{1 *}$, S. Dharanya ${ }^{1}$, S. Shaheen ${ }^{1}$ and A. Umamakeswari ${ }^{2}$ \\ ${ }^{1}$ M.Tech Embedded Systems, School of Computing, SASTRA University, Thanjavur, Tamil Nadu, India; \\ divyavijay.ece11@gmail.com, dharanyasubramanian@gmail.com, shaheenece@gmail.com \\ ${ }^{2}$ Associate Dean, School of Computing, SASTRA University, Thanjavur, Tamil Nadu, India;
}

aum@cse.sastra.edu

\begin{abstract}
Robots have been widely used to perform variety of tasks which reduces the manual work specifically in remote areas where human accessibility is unimaginable. The main applications where the robots have exhibited their excellence include surveillance, tracking targets for military purposes and also for disaster management like searching and rescuing victims. This paper deals about an evolutionary Non-humanoid robot for surveillance with intruder protection capability. The unique feature of this surveillance robot is that it can travel both on land and water and is equipped with robotic arm to handle the work safely. It is armed with a low power high performance NXP processor which is able to manage power more efficiently. Besides being able to work in Semi-autonomous mode, the CCD (Charge Coupled Devices) along with camera aids the operator to control it in manual mode.
\end{abstract}

Keywords: Amphibious, CCD, NXP processor, Robots, Self-defending, Surveillance.

\section{Introduction}

A robot is a machine capable of replicating or resembling the human actions with the collection of components like sensors, power supplies and controls. The essential characteristics of the robot are Sensing, Movement, Energy, and Intelligence [4]. These characteristics have enabled them to replace the human beings in many critical situations. The high accuracy and precision in their work make them more efficient than the human beings.

Surveillance is a process of continuously monitoring an area/field to gather sufficient information about the type and nature of the surrounding environment. There are many types like area surveillance for security purposes, surveillance on an individual or a group for monitoring their behaviour and surveillance for monitoring the traffic violations. All these processes need huge manpower and extreme caution in case of any surreptitious issue as surveillance is very difficult aspect in this world. A surveillance robot can be very useful in these cases in which human intervention involves potential threat. It is an unmanned vehicle which can traverse on many varieties of surfaces like flat or rocky land terrains and water. Some robots capable of traversing on both land and water are of great use in military surveillance. One such amphibious robot is designed in this work.

\section{Existing System}

Many different robots have been developed with different cause and aim. Recent innovations include an autonomous terrain robot which can navigate on flat floors and climb staircases with Position sensitive detectors (PSD) being used to estimate the angle of the obstacle. Adaptive driving mode is used in transition between the modes [12]. A mobile robot that can climb and descend stairs with the help of sensors was also designed. The centre of gravity (COG) and

\footnotetext{
* Corresponding author:

V. Divya (divyavijay.ece11@gmail.com)
} 
the height of the steps are measured [9]. Robots have also been used for home surveillance like the jumping robot developed for indoor security system enabled with zigbee network of wireless PIR (Passive InfraRed) sensor nodes [7]. A method to track a person inside a room have been introduced which is based on a mobile robot using HPPH (horizontal-projecting probability histogram) method to recognize the person [3].

Humanoid robots are a great attraction recently. LOLA is a humanoid robot which is stable and autonomous. It can walk like humans with its 7-DoF leg design [10]. Another humanoid robot which can assist the elderly people was designed with an aim to work in real time environments like home and hospital. The robot used visual as well as LRF (Laser Range Finder) sensors to navigate around the area [13].

More recently robots have been used for surveillance on both land or on water with an omni-directional tracking model. [2]. A surveillance coverage scheme for flying robots to be used in inaccessible areas was introduced. Initially they employed a single robot to find out the route using monocular vision. Once the map has been generated, it assigns the map to the following robots so that the coverage efficiency is high [8]. An Amphibious robot to study about the marine habitats using IMAPS2 (Interactive Mobile Amphibious Probing and Surveillance System) was designed [11]. A flipper mechanism for moving the unmanned navigator over uneven terrains was proposed in [1]. There are many surveillance robots efficiently used in real time environments. The following describes two of them.

\subsection{Cougar 20-H}

Cougar $20-\mathrm{H}$ is a surveillance robot developed for military applications with the ability to sense through the walls (STTW) and also to detect the breathing state of the target. It is an on land surveillance robot which can detect the targets within buildings or it let the fire fighters to locate the people inside the buildings on fire. Multiple integrated cameras help them to have day as well as night time vision. The robot being lightweight can travel swiftly with its caterpillar tracks. It can be easily operated from a PC which is more than 300 feet away. The RF transceiver placed in the robot helps to send and receive high energy signals which can easily pass through the wide walls. Reflections from the targets are received and processed to locate the targets [5].

\subsection{Sandia Fire Ant Robot}

Sandia developed a mobile robot that can launch projectile named Fire Ant. The missile used in this robot is named as EFP (Explosive Formed Projectile). This robot was Sandia's first robot which uses an autonomous stand-off mine to detect the mines and diffuse it. It also marks the cleared off areas in the field, helping the people to walk-through. Dixie, one of the sandia's robot acts as the base for this Fire ant robot. The video motion detection selects the target direction; once the direction has been pointed the operator can track the path. The robot destroys itself along with the firing of projectile [6].

\section{Proposed Model}

The proposed model is an all terrain surveillance robot with smart nodal sensors. This is a non humanoid robot for surveillance in the military as well as in domestic side. It has combined features of cougar 20- $\mathrm{H}$ (capable to survive on land) and fire ant (capable to survive on water). It is very studious with its capabilities of surveillance through a powerful camera. During surveillance, normal spy bots are caught by the strangers and destroyed easily. But this model has self defending technology, which has the power of self guarding on its own. It can be controlled manually through RF communication. A semi autonomous mode is also available in the model.

It has robotic arm to lift light weight objects as well as to cut feeble wires in the time bombs. It really looks like human hand and possess four movements. Its unique implementation with a caterpillar track makes it possible to travel in crucial conditions and rocky regions. The whole model is built on a floating board to enable it to move on water. The remote surveillance from the PC makes it easy to handle and the sensors such as proximity and temperature makes it smarter as the name itself suggests it. The ARM7 TDMI is used as main controller for sensor control which provides greater performance and low power utilization.

\section{Block Diagram}

The block diagram of the non-humanoid robot is as shown in the Figure 1, which consists of three sections: power unit, peripheral interface unit and the remote monitoring unit. 


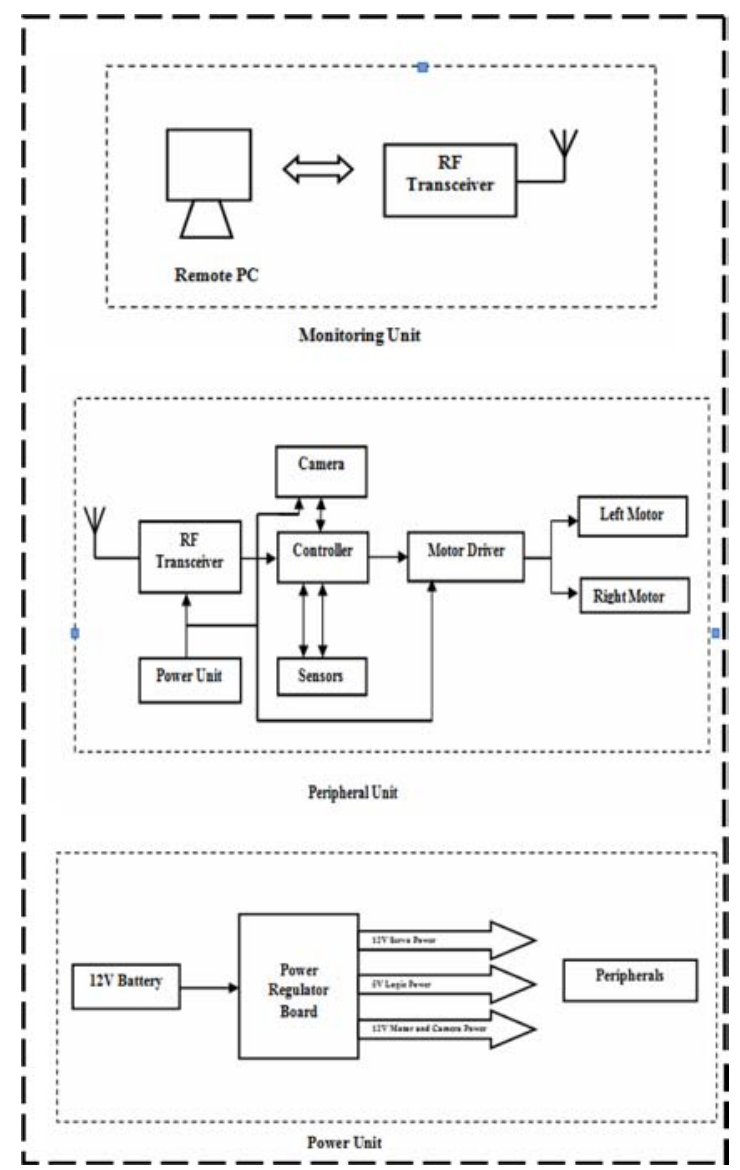

Figure 1. Block diagram of the entire system.

\subsection{Power Unit}

A $12 \mathrm{~V}$ battery unit is used as main source of power which is regulated to provide $5 \mathrm{~V}$ power for the peripherals like motors, cameras, sensors and RF unit.

\subsection{Peripheral Unit}

The main ARM controller is interfaced with peripheral drivers to control the motors (right and left), different sensors like ultrasonic sensor, optical sensors, accelerometer and camera units. Additionally it is interfaced with RF trans-receiver unit to communicate the sensed values to the remote PC and to receive the control commands.

\subsection{Monitoring Unit}

This unit consists of a remote computer and a RF communication unit to monitor and control the robot. The user can give the control commands through PC to move the robot manually and see the surveillance results on the PC screen.

\section{Results}

The Figure 2 shows the Emulation of LPC2148 done utilizing KEIL micro version debuggers from KEIL micro system. The program is written in the debugger and compiled for errors. Once the debugging and glitch finding is finished, the project is cross compiled and the HEX file is created. The Hex is of Intel hex standard.

The program in the debugger is shown in the Figure 3. After the Hex compilation, the simulation procedure starts. The simulation is verified by various general instructions and inputs. The simulated system is thus run through to find the glitches if any and the final software is produced for downloading it to the target systems. This procedure followed is shown in Figure 4. Figure 5 shows the hardware setup of the whole robot.

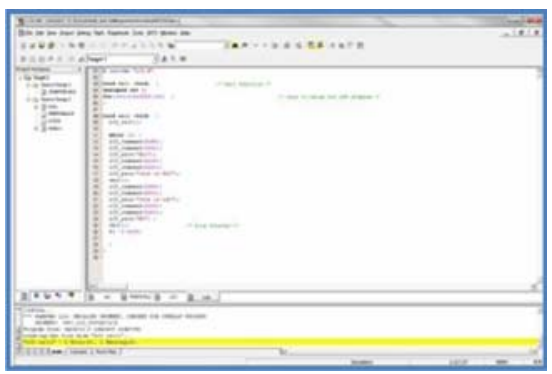

Figure 2. Emulation.

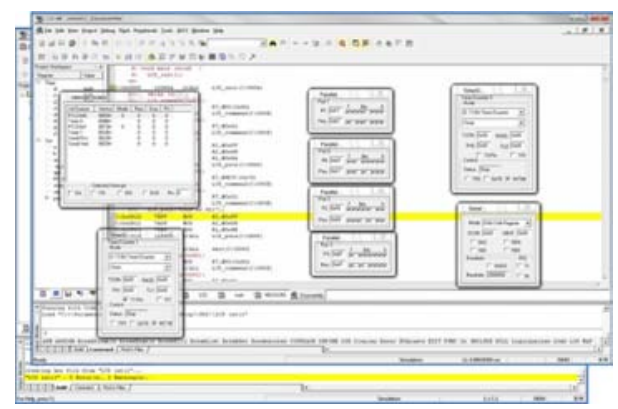

Figure 3. Compilation.

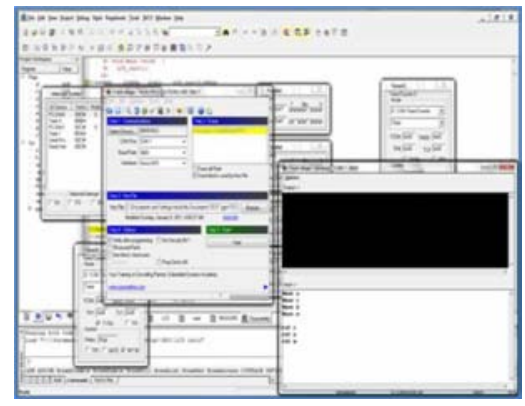

Figure 4. Simulation. 


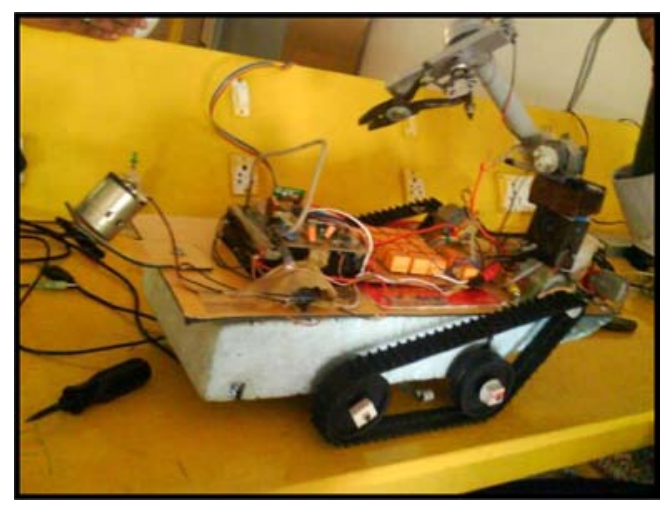

Figure 5. Hardware implementation of the entire system.

\section{Conclusion}

A non humanoid robot which can survive both on land and on water is designed with the ability to safeguard itself from enemies. In addition, it can diffuse the bomb with the help of robotic arm fitted in it. And the result shows that the system meets the given necessities of the task. The model could be used to form an army of robots useful in surveillance and rescue operation. In near future, the robots will play a vital role for future developments in surveillance technologies and in saving the precious human life.

\section{References}

1. Mohebbi A, Safaee S et al. (2010). Design, simulation and manufacturing of a tracked surveillance unmanned ground vehicle, Proceedings of the IEEE International Conference on Robotics and Biomimetics, 1268-1275.

2. Boult T E, Gaob X et al. (2004). Omni-directional visual surveillance, Image and Vision Computing, vol 22, 515-534.
3. Hu C, Ma X et al. (2010). Reliable people tracking approach for mobile robot in indoor environments, Robotics and Computer-Integrated Manufacturing, vol 26, 174-179.

4. Cougar $20 \mathrm{H}$ Sees through walls. Available from: http:// www.darkgovernment.com/news/cougar-20h-seesthrough-walls.

5. Fire Ant, Available from: http://robotics.sandia.gov/fireant. html.

6. Introduction to robots. Available from: http://www.galileo. org/robotics/intro.html.

7. Zhang J, Song G et al. (2011). An indoor security system with a jumping robot as the surveillance terminal, IEEE Transactions on Consumer Electronics, vol 57( 4), 1774-1781.

8. Doitsidis L, Weiss S et al. (2012). Optimal surveillance coverage for teams of micro aerial vehicles in GPS-denied environments using onboard vision, Autonomous Robot Journal, vol 33(1-2), 173-188.

9. BenTzvi P, Ito $S$ (2009). A mobile robot with autonomous climbing and descending of stairs, Robotica Journal, vol 27(2), 171-188.

10. Buschmann T, Lohmeier S (2009). Humanoid robot Lola: Design and walking control, Journal of Physiology, vol 103(3-5), 141-148.

11. Tang Y, and Zhang H (2008). An interactive mobile amphibious probing and surveillance system, Proceedings of the IEEE International Conference on Information and Automation (ICIA), 441-445.

12. Kim Y, Kwak J et al. ( 2012). An autonomous terrain adaptation and user-friendly tele-operation of wheel-track hybrid mobile robot, International Journal of Precision Engineering and Manufacturing, vol 13(10), 1781-1788.

13. Mohamed Z, and Capi G (2012). Development of a new mobile humanoid robot for assisting elderly people, International Symposium on Robotics and Intelligent Sensors (IRIS), Procedia Engineering, vol 41, 345-351. 